\title{
Deficiency of Standard Effective-Medium Approximation for Ellipsometry of Layers of Nanoparticles
}

\author{
E. G. Bortchagovsky, ${ }^{1,2}$ A. Dejneka, ${ }^{3}$ L. Jastrabik, ${ }^{3}$ V. Z. Lozovski, ${ }^{2}$ and T. O. Mishakova ${ }^{2}$ \\ ${ }^{1}$ Institute of Semiconductor Physics, NASU, pr. Nauki 41, 03028 Kiev, Ukraine \\ ${ }^{2}$ Institute of High Technologies, Taras Shevchenko National University of Kiev, pr. Glushkova 4g, 03033 Kiev, Ukraine \\ ${ }^{3}$ Institute of Physics of the AV ČR, Na Slovance 2, 18221 Praha 8, Czech Republic
}

Correspondence should be addressed to E. G. Bortchagovsky; bortch@yahoo.com

Received 16 September 2015; Accepted 18 November 2015

Academic Editor: Mohamed Bououdina

Copyright (C) 2015 E. G. Bortchagovsky et al. This is an open access article distributed under the Creative Commons Attribution License, which permits unrestricted use, distribution, and reproduction in any medium, provided the original work is properly cited.

\begin{abstract}
Correct description of optical properties of layers of disordered interacting nanoparticles is the problem. Contrary to volumes of nanocomposites, when standard models of effective-medium approximations (EMA) work well, two-dimensional case of layers has intrinsic anisotropy, which influences interparticle interactions. The deficiency of standard Maxwell-Garnett model in the application to the ellipsometry of layers of gold nanoparticles is demonstrated. It demands the modification of EMA models and one way of this is considered in this paper. Contrary to existing 2D models with phenomenological parameters, the proposed Green function approach uses the same number of parameters as standard 3D EMA models for explicit calculations of effective parameters of layers of disordered nanoparticles.
\end{abstract}

\section{Introduction}

Peculiar properties of composites comprising metallic nanoparticles attracted attention for millenniums and have been exploited in the production of fascinating art pieces like the Lycurgus cup, stainless glasses, Deruta ceramics, and so forth. Properties of composites found the explanation in the effective-medium approximation (EMA) models, which in the optical case define effective dielectric function from averaged fields according to the expression $\langle D\rangle=\langle\varepsilon E\rangle=\varepsilon_{\text {eff }}\langle E\rangle$. First of those models was developed already in the end of the 19th century [1]. Up to now, those models, namely, Maxwell-Garnett (MG) and self-consistent Bruggeman ones, are the most popular in the description of optical properties of nanocomposites. Although very little information about the composite is included into those models, their robustness is defined by restrictive bounds for the possible resulting dielectric function [2].

These models are also widely used for layers of nanoparticles schematically shown in Figure 1 . However, as these models are based on the Lorentz sphere formalism, their application to such layers creates some problems as already reported in literature [3-5]. The reason is that the planar geometry of the layer gives obvious geometrical anisotropy along and across the layer. As a result, electromagnetic interparticle interactions along and across the layer are different [6] as shown in Figure 2. In a three-dimensional case, we should expect two more neighbor dipoles with repulsive interactions resulting in zero sum field from all neighbors at the position of the central particle. This is the meaning of the Lorentz sphere formalism of averaged field for regular lattices as well as for random uniform distribution of particles. However, for the layer the average field from neighbor dipoles is not zero. In the case of the longitudinal polarization, the total interaction is attractive in average resulting in a redshift of the nanoparticle resonance, but for the transverse polarization the interaction is always repulsive which results in the blueshift of the resonance [6]. Such a difference splits the resonance of $3 \mathrm{D}$ composite into two resonances for 2D layer of nanoparticles.

In fact, more detailed account of even only dipolar interparticle interactions in the case of randomly distributed inclusions in 3D composite indicates some deviation of the local field from zero $[7,8]$. It is so called fluctuations of 


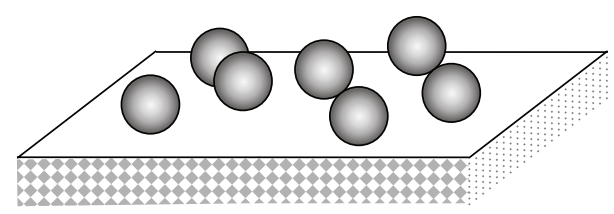

FIGURE 1: System of nanoparticles randomly distributed on a surface.

the field due to irregular distribution of inclusions [8]. It is not surprise that the fluctuation of the local field in 2D composite is remarkably bigger than in the case of $3 \mathrm{D}$ composite [9] in addition to the nonzero average field in this case [6].

Expressions for standard noticed EMA models may be written in a common way [2] as the weighted sum of polarizabilities of all components calculated via the ClausiusMossotti expression:

$$
\frac{\varepsilon_{\mathrm{eff}}-\varepsilon_{h}}{\varepsilon_{\mathrm{eff}}+y \varepsilon_{h}}=\sum_{i} q_{i} \frac{\varepsilon_{i}-\varepsilon_{h}}{\varepsilon_{i}+y \varepsilon_{h}} \quad \text { with } \sum_{i} q_{i}=1,
$$

where $\varepsilon_{i}$ are dielectric functions of constituting $i$ materials, $\varepsilon_{h}$ is the one for the host material, which embeds all other ones, $q_{i}$ are the volume fractions, and depolarization factor $y=(1-l) / l$ with $l$ being the screening parameter.

The Bruggeman model in spite of its higher precision in comparison to the MG one does not reproduce the plasmon resonance of nanoparticles in the composite $[10,11]$, as localized plasmons are so much restricted in space by the particle local environment that they do not feel the properties of the whole composite. Thus we shall restrict our further consideration only to the MG model. It results in the case of only two materials with $\varepsilon_{h}=\varepsilon_{1}$ and spherical $(l=1 / 3$ or $y=2$ ) inclusions

$$
\begin{aligned}
\varepsilon_{\mathrm{eff}} & =\varepsilon_{1} \frac{\varepsilon_{2}\left[\left(1+2 q_{2}\right) /\left(1-q_{2}\right)\right]+2 \varepsilon_{1}}{\varepsilon_{2}+\left[\left(2+q_{2}\right) /\left(1-q_{2}\right)\right] \varepsilon_{1}} \\
& =\varepsilon_{1}\left[1+\frac{3 q_{2}}{1-q_{2}} \frac{\varepsilon_{2}-\varepsilon_{1}}{\varepsilon_{2}+\left[\left(2+q_{2}\right) /\left(1-q_{2}\right)\right] \varepsilon_{1}}\right] .
\end{aligned}
$$

If one sphere has dipolar plasmonic resonance at $\varepsilon_{2}=$ $-2 \varepsilon_{1}$, MG model for Drude metal inclusions redshifts this resonance to

$$
\varepsilon_{2}=-\left(2+\frac{3 q_{2}}{1-q_{2}}\right) \varepsilon_{1} .
$$

The reason of this shift is the additional polarization of the host by long-range components of the field of inclusions. In the case of layers, this long-range polarization is accompanied by additional nonzero direct interparticle interactions which modifies the shift too. At the same time the standard EMA expression is only a sum of polarizabilities of individual inclusions with no account of direct interparticle interactions. And if the lateral interactions can be resembled to some extent by the MG model, it is impossible to reproduce by any standard EMA models a blueshift generated by transverse interactions. Weak sensitivity of ellipsometry to transverse optical anisotropy of films [12] hides that resonance in ellipsometric spectra but can not approve the application of incorrect models, at least without clear understanding of their deficiencies.

Correctly taking into account anisotropic interactions in layers is not difficult in the case of a regular distribution of identical particles in nets of some lattice [4, 13-16]. However, if one or more parameters exhibit random variances, the problem is less tractable. The main difficulty even in the case of equal size and shape of all inclusions is the necessity to average the final results which depend on the concrete topology of the composite. In many cases it is substituted by the calculations for the averaged parameters. Approaches with the averaging on the orientation [17] or shape [18] were successfully made for $3 \mathrm{D}$ systems but for $2 \mathrm{D}$ layer of nanoparticles authors are loose usually themselves in averaging [19]. And definitely standard approach applied to the transverse component of the effective dielectric function [19] does not reproduce discussed repulsive interparticle interaction and resulting blueshift of the resonance.

Standard EMA models have other deficiencies, namely, general consideration in the Rayleigh approximation of all inclusions as small objects possessing dielectric function of bulk materials and specifically for layers-no account of the interaction of particles with a substrate. However, different approaches were proposed to account these deviations. Gustav Mie has built the model for the account of the change of optical properties of a particle with increased size [20] and the use of the general Mie expression for the polarizability in EMA was proposed for not small particles [21, 22]. From the other side, the change of the dielectric function for small particles due to the scattering of electrons by surface [23] or due to approaching quantum confinement for tiny particles [24] was considered and the modified dielectric function (at least by the former mechanism) is used in a number of investigations. The account of the modification of the polarizability of particles deposited on a surface by their interaction with the surface in the frame of the interaction with the mirror image is rather straightforward and also was proposed for the modification of MG model for layers [4, 15]. Even the account of the finite size of particles interacting with their images was made in a recurrent manner [25].

For the sake of complexity of this consideration of restrictions of standard EMA models and approaches to the description of optical properties of disordered layers of nanoparticles, it is necessary to notice that more complex EMA models like [26] including in the first place topological effects exist. For layers of nanoparticles, the solution given by Bedeaux and Vlieger [27] may be considered as the most completed approach. However the complex mathematics connecting physical parameters of the system like size and concentration of nanoparticles with optical properties of the layer makes this model less applied.

Even the first approach for the description of optical properties of layers of ordered nanoparticles [13-16] included all interactions into depolarizing factor, which became one additional fitting parameter. However those approaches do not give the way to determine these parameters from size (polarizability) and concentration of nanoparticles for disordered systems. More sophisticated theory [27] with very 


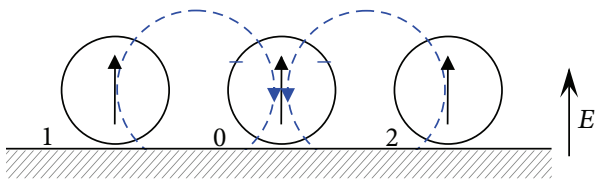

(a)

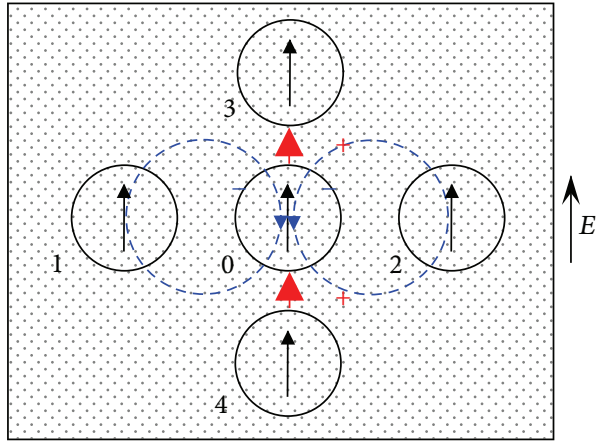

(b)

FIGURE 2: Scheme of interactions of particles on a surface excited perpendicularly (a) and along (b) the surface.

cumbersome way of such calculations exchanges it by the introduction of phenomenological parameters accounting interactions. From this point of view, those approaches are not different from artificial addition of few Lorentz resonances to some background dielectric function for the reproduction of optical properties of a layer of nanoparticles $[28,29]$.

\section{Experimental Ellipsometric Investigations}

Spectroscopic ellipsometry of layers of gold nanoparticles was performed by Woollam's WVASE device. Samples were prepared by thermal evaporation of gold on a glass substrate and annealing at $250^{\circ} \mathrm{C}$ for 2.5 hours. Gold was deposited as a wedge with the variation of the mass thickness from about 2 to $25 \mathrm{~nm}$.

Annealing of metal films and surface diffusion were widely investigated (e.g., [30]). Creation of small separated metallic particles with rather regular shape from thin deposited films can be done either by long-term low temperature annealing as in this work or by fast high temperature flash annealing [31]. Films of very small thickness deposited by thermal evaporation consist of small islands. It may be seen in Figure 3(a) with spectra for the thinnest, of about 1-2 nm deposited film, where only one weak broad feature of about $2.25 \mathrm{eV}$ indicates the presence of plasmon excitation on islands. The bend over $4 \mathrm{eV}$ corresponds to the beginning of the glass absorption. Figure $3(\mathrm{~b})$ demonstrates similar spectra for thickest, of about $25 \mathrm{~nm}$, deposited film. Many features correspond to complex structure of that film, which definitely does not consist of only separated islands. However after annealing that film demonstrates only one feature of localized plasmons on separated particles, as seen in Figure 3(c).

It is necessary to say that sphere and spheroid are not economical forms. Let us assume that annealing of a completed film of the thickness $d$ produces dense monolayer of identical spheroids with the rotation axes perpendicular to the surface. If we denote the radius of these spheroids by $R$ and the half of its third axis perpendicular to the surface by $b$, the thickness of the resulting film will be $2 b$, and the area occupied by each spheroid is $2 \sqrt{ } 3 R^{2}$ in the case of hexagonal packing and $4 R^{2}$ for square packing. It means that filling fraction of the surface is 0.907 or 0.785 for these lattices correspondingly. Even if this spheroid is inscribed into a cylinder, volume fraction of such a structure is $2 / 3$ and for considered lattices this value should be multiplied by the filling fraction. To consider the material balance, for the hexagonal lattice $2 \sqrt{ } 3 R^{2} d=4 / 3 \pi R^{2} b$ or $b=(3 \sqrt{3} / 2 \pi) d \approx 0.827 d$; that is, the thickness of the resulting film is 1.65 of the initial one. For the square lattice, this value is 1.91. It is interesting to note that if we consider the annealed film consisting of semispheroidal protrusions, their vertical size will be equal to the vertical size of spheroids, so the thickness of the annealed film will be the same. If we have not completed coverage, corresponding filling fraction should be included into the material balance. For example, if we consider particles as spheres, annealing of a film of the thickness of $24 \mathrm{~nm}$ would produce dense packing of particles with the radius of about $20 \mathrm{~nm}$ or $32 \mathrm{~nm}$ if the filling fraction is 0.25 . In reality, the spreading of sizes of particles produced by thermal annealing is rather wide.

Results for the area with the mass thickness of about $15-20 \mathrm{~nm}$ (sample N1) and their fitting by the standard 3D MG model are presented in Figure 4. Parameters of the glass substrate were determined beforehand from measurements for a clean area; gold parameters (not Palik) were used from the WVASE software (J. A. Woollam Co., Inc. [32]) and particles were considered as situated in air. There was the proposal to account the interaction of particles with the substrate by embedding them into some medium with new dielectric function [33], but in fact it is only the attempt to account interactions in the system by an additional fitting parameter. Similar results may be obtained fitting the depolarizing factor, which in such a case includes also results of different interactions [14-16]. The approach with new dielectric function is rather artificial and may be approved only if particles are embedded partially into the substrate. In the present case, only the volume fraction of gold, which corresponds to the filling fraction of the surface in the case of the monolayer of particles, and the thickness of the film were used as fitting parameters.

Results of the fitting are given in Table 1 for all samples assuming particles are spherical. Samples with smaller number have bigger amount of the deposited gold. The first 


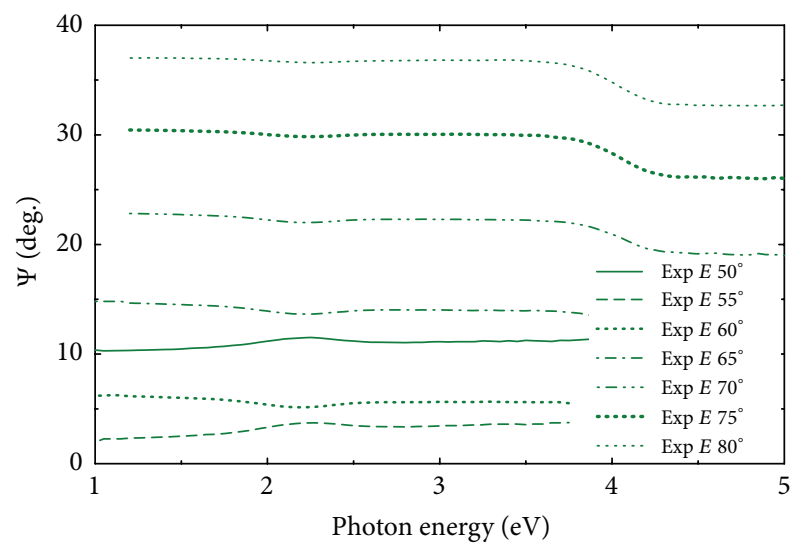

(a)

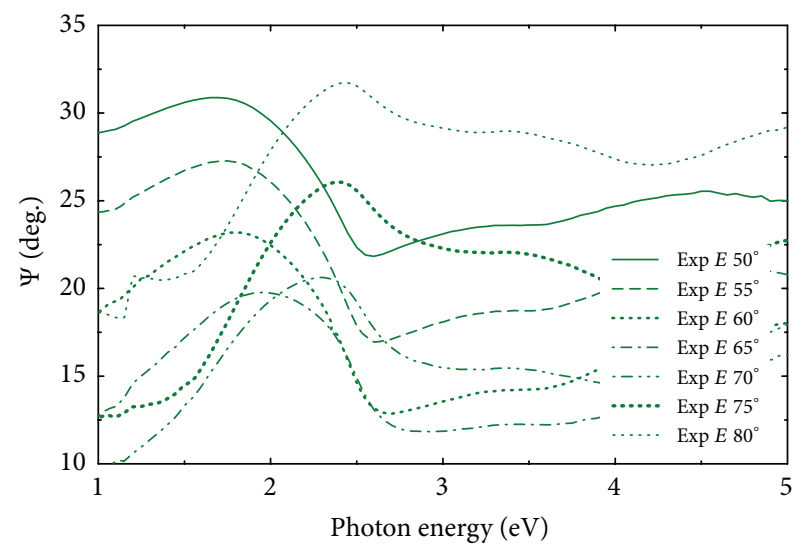

(b)

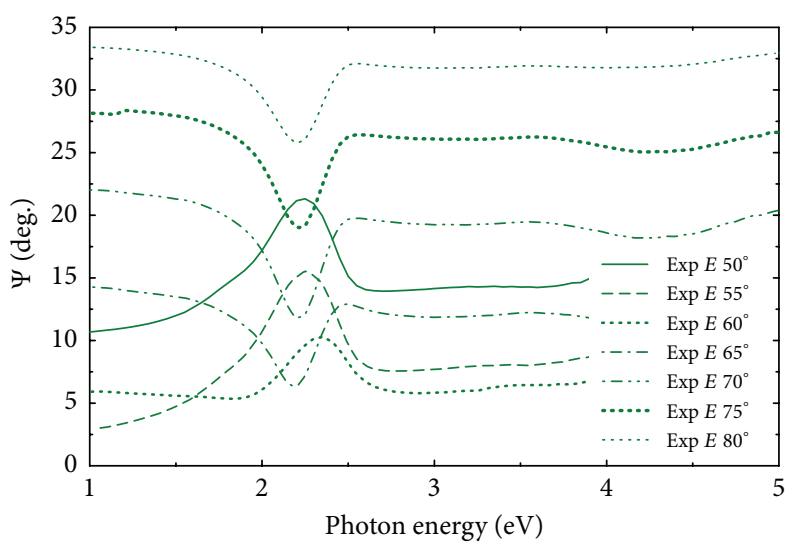

(c)

FIGURE 3: Spectra of the angle $\Psi$ of (a) thinnest deposited film (sample N8 with the gold mass thickness of about $1 \mathrm{~nm}$ ); (b) thickest deposited film; and (c) annealed thickest film (sample N0 with the gold mass thickness of about $25 \mathrm{~nm}$ ).

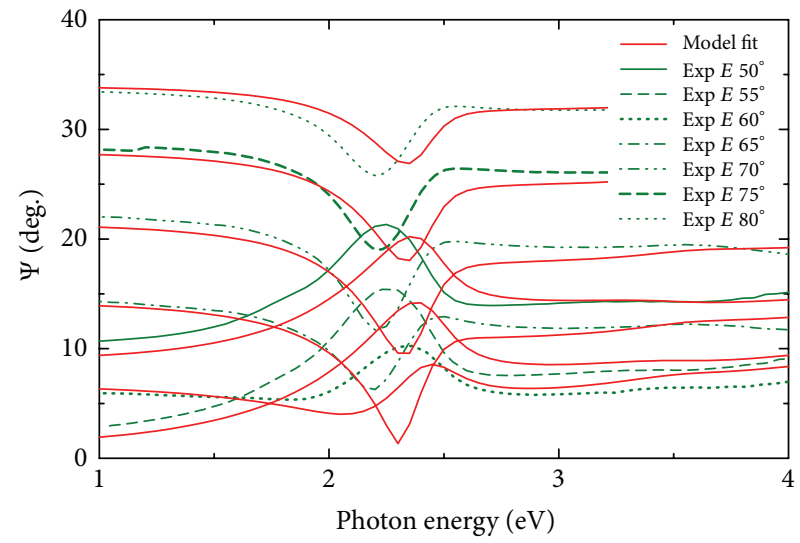

(a)

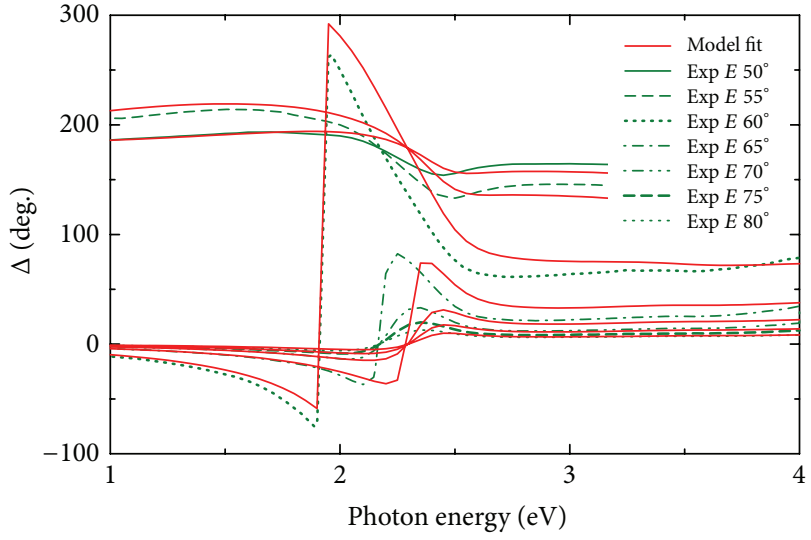

(b)

FIGURE 4: Ellipsometric spectra with the best fit by Maxwell-Garnett model of the sample with the mass thickness of gold about $20 \mathrm{~nm}$.

problem is the visible in Figure 4 blueshift of resonance in the model compared to the experimental one, which exists for all thicknesses. The fact that this discrepancy exists even for samples with the smallest amount of gold (few nanometers of the mass thickness what corresponds to the size of produced nanoparticles less than tens of nanometers) indicates that size effect is not responsible for the discrepancy. This demonstrates that the model does not account direct interparticle interactions to reproduce the whole observed redshift. The use of fitted resonance to avoid this deficiency of EMA models $[28,29]$ can not be considered as the solution of the problem. 
TABLE 1: Results of fitting by the Maxwell-Garnett model.

\begin{tabular}{lccc}
\hline Sample number & $\begin{array}{c}\text { Thickness } \\
d, \mathrm{~nm}\end{array}$ & $\begin{array}{c}\text { Volume fraction } \\
q,(\%)\end{array}$ & $\begin{array}{c}\text { Mass thickness } \\
d \times q, \mathrm{~nm}\end{array}$ \\
\hline 0 & 79.868 & 32.133 & 25.664 \\
1 & 45.705 & 29.837 & 13.637 \\
2 & 31.340 & 29.155 & 9.137 \\
3 & 14.391 & 28.189 & 4.057 \\
4 & 11.461 & 27.487 & 3.150 \\
5 & 8.410 & 26.478 & 2.227 \\
6 & 6.885 & 26.212 & 1.805 \\
7 & 5.791 & 25.682 & 1.487 \\
8 & 3.786 & 25.436 & 0.963 \\
\hline
\end{tabular}

If the depolarizing factor $y$ is also fit, it decreases for samples with big mass thickness from $1 / 3$ to $0.21-0.25$ corresponding to oblate particles with an eccentricity of 22.7. It is reasonable result as annealing of films with bigger mass thickness of deposited gold produces bigger separated particles of not spherical but more flattened form. This correction of the eccentricity redshifts resonances, but it does not improve the fit noticeably.

A less obvious problem is the determined volume fraction of gold for all those films in the range of $25 \%-32 \%$. It is beyond the formal range of validity of the MG model. If to use noticed features of spheroidal form that even been inscribed into a cylinder, it gives the volume fraction only $2 / 3$, fitted values correspond to filling fraction of the surface about $0.4-0.45$. At the same time, the resulting mass thickness has reasonable values.

Figure 5 demonstrates the position of the feature corresponding to the localized plasmon resonance (here minimums and maximums of the experimental spectra of $\Psi$ ) versus the angle of incidence and the amount of gold, which directly corresponds to the filling fraction of the surface by particles. The closer the particles, the bigger the interaction. The bigger the interaction, the larger the redshift of the resonance. The observed redshift of the resonance clearly indicates that we monitor the longitudinal one. The shift of the recorded feature with the angle of incidence was observed previously [14] and its reason is the angular dependence of the phase shift of the total surface field exciting nanoparticles [34]. Just that angular dependence of the phase shift results in the arising of a wave-like structure in the experimental spectra of $\Psi$ around the Brewster angle where the resonance position coincides with neither minimum nor maximum of the structure [34]. As a result, as indicated in Figure 5, position of extremums demonstrates wave-like structure around the incidence of $60^{\circ}$ for thickest films (about and more than $10 \mathrm{~nm}$ of mass thickness according to results presented in Table 1). However the main feature of Figure 5 is the pronounced redshift of the resonance at increasing of the amount of deposited gold. To demonstrate that size effect (variation of the diameter of Au spherical nanoparticles from few to $50 \mathrm{~nm}$ redshifts the resonance for $\sim 0.1 \mathrm{eV}$ ) is not solely responsible for this shift, let us consider what we can get from the MG model in more detail.

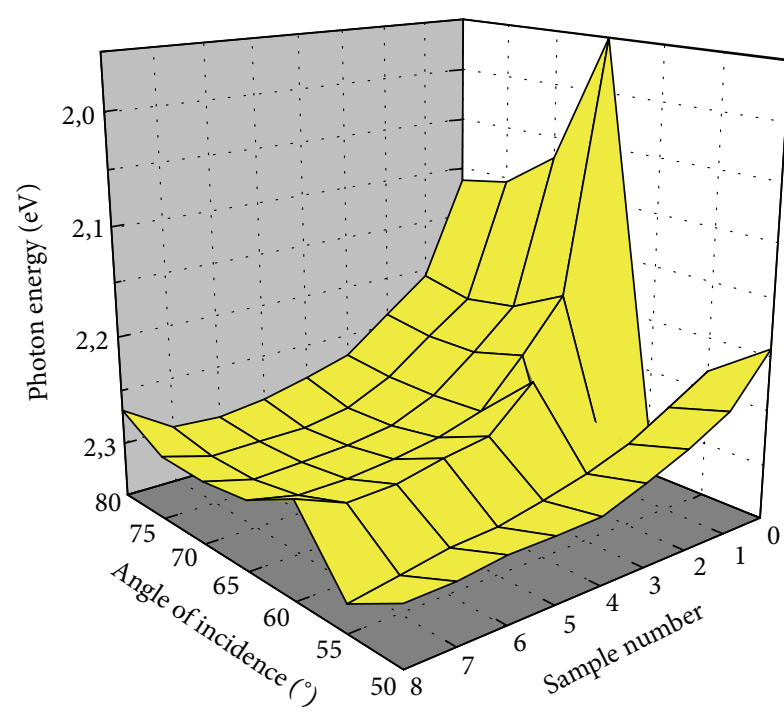

FIgURE 5: Behavior of the visible position of the localized plasmonic resonance versus the angle of incidence and the amount of deposited gold ( 0 corresponds to the biggest and 8 to the smallest amount of deposited gold).

The influence of the MG model can be considered as the modification of the screening parameter $l$ to the effective value $l(1-q)$. The volume fraction $\sim 0.3$ shifts the resonance of separated gold spherical particles from $\sim 2.48 \mathrm{eV}$ to $\sim 2.42 \mathrm{eV}$ (both values are modified by relatively high dissipation of gold; without the dissipation they would be $\sim 2.67 \mathrm{eV}$ and $\sim 2.48 \mathrm{eV}$ ). For oblate particles taking even the limiting value of the screening parameter of 0.22 obtained at fitting of the thickest film with $\sim 25 \mathrm{~nm}$ of the mass thickness of gold (eccentricity $e=2.5$ ) and a volume fraction 0.25 obtained at fitting of thinnest films with 1-2 nm of mass thickness of gold (for this thickness even fitting with variable screening factor left its value at $\sim 1 / 3$ as for sphere), the resonance position calculated by the MG modes is about $2.33 \mathrm{eV}$, but the recorded resonance even for the smallest amount of gold is at $\sim 2.26 \mathrm{eV}$ as shown in Figure 5.

\section{Local Field Approach to the Effective Polarizability of Layers of Nanoparticles}

The problem in the calculation of the response of a complex system is that we know the local polarizability $\chi$ of the constituting parts and the external exciting field $\mathbf{E}^{(\mathbf{0})}$, but the local response is defined by the local field $\mathbf{E}$. To use the external field we have to introduce the new polarizability $\mathbf{X}$ accounting the influence of the whole system to fulfill

$$
\mathbf{P}=\chi \mathbf{E}=\mathbf{X E}^{(0)}
$$

Having many nanoparticles on a surface, we can write the Lippmann-Schwinger equation for the total field at one of them at a point $\vec{R}$ which is the sum of the external exciting fields with the frequency $\omega$ and calculated via the Green 


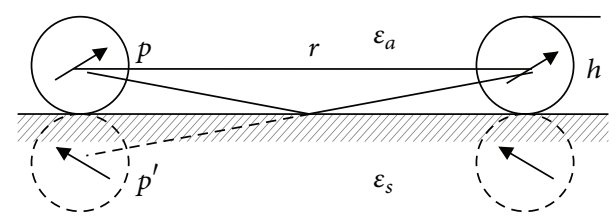

FIGURE 6: Scheme of particles self-action due to the image generation and interparticle interactions both directly and via the substrate.

function the field generated by all other particles situated at points $\vec{R}^{\prime}$ :

$$
\begin{aligned}
E_{i}(\vec{R}, \omega) & \\
= & E_{i}^{(0)}(\vec{R}, \omega) \\
& \quad-k_{0}^{2} \sum_{\alpha} \int_{V_{\alpha}} d \vec{R}^{\prime} G_{i j}\left(\vec{R}, \vec{R}^{\prime}, \omega\right) \chi_{j l}(\omega) E_{l}\left(\vec{R}^{\prime}, \omega\right)
\end{aligned}
$$

with the integration over the volume of particles.

However, considering particles small enough to work in the dipole approximation, integration can be omitted and the Green $G_{i j}$ function will depend only on the difference of the coordinates of interacting particles. This function takes into account both direct interactions and indirect ones via the field reflected from the surface as shown in Figure 6. The initial polarizability of the particle $\chi_{i j}$ is taken with the account of the self-action by the image in the surface, finite size, and so forth to avoid the necessity to deal with the field in the source [5].

The restriction of the consideration by the dipole approximation demands rather separated particles with the distance between them of the order of the size of particles. However, this restriction is satisfied up to the filling fraction of $30 \% \div$ $40 \%$ that is much higher than the restriction of MG model. Such a value approaches the percolation threshold. Even some higher value restricting the validity of the dipole approximation was given in [27].

The problem can be considered both in the direct $r$-space and in $k$-space by the Fourier transformation. The former approach may be clearer sometime but produces oscillating sums and integrals like Fresnel ones with bad convergence. This problem is absent in $k$-space where, additionally, all integral convolutions turn to multiplications [35-37]. As we consider all particles as point dipoles dispersed over the surface at the constant distance, it is convenient to work in the so-called $k-z$ representation with the Fourier transformation only in the $2 \mathrm{D}$ surface plane.

For the random distribution of these particles on a surface, we have to perform averaging. It may be written for the random uniform distribution as

$$
\begin{aligned}
& \overline{\sum_{a=1}^{N} G_{i j}\left(\vec{r}-\vec{r}_{a}, z, z_{a}, \omega\right) \chi_{j l}(\omega) E_{l}\left(\vec{r}_{a}, z_{a}, \omega\right)} \\
& \quad=n \int \frac{d \vec{k}}{(2 \pi)^{2}} e^{-i \vec{k} \vec{r}} G_{i j}\left(\vec{k}, z, z_{a}, \omega\right) \chi_{j l}(\omega) E_{l}\left(\vec{k}, z_{a}, \omega\right),
\end{aligned}
$$

where $n$ is the concentration of particles on the surface and $\vec{r}$ and $z$ are in-plane and perpendicular coordinates correspondingly. Making the Fourier transformation of the expression for the dipole moment and using some algebra [36], we can obtain finally

$$
\begin{aligned}
X_{j i} & \left(\vec{k}_{\|}, z_{\alpha}, \omega\right) \\
\quad= & {\left[\left(\tilde{\chi}_{i j}(\omega)\right)^{-1}+n k_{0}^{2} G_{i j}\left(\vec{k}_{\|}, z_{\alpha}, z_{\alpha}, \omega\right)\right]^{-1}, }
\end{aligned}
$$

where $\vec{k}_{\|}$is the component of the wave vector along the layer. It is worth noticing that these expressions are the limiting case for the constant binary correlation function of the particle distribution, which simplifies calculations. Formally expression (6) can be used for any binary correlation function applied at the averaging.

Expression (7) has the standard form of the correction of the response by an additional interaction, which is described in our case via the Green function.

If we consider the external exciting field as the plane wave, its Fourier image is delta-function and the inverted transformation is trivial allowing using this expression for the total averaged polarizability of one particle. However, as we average values for random particle distribution, we should not work with it directly but have to multiply it by the concentration $n$ to receive the polarizability of the unit area of the film $[36,37]$.

All interactions modifying the polarizability can be included into the effective screening parameter [14-16]. It should be used to calculate the initial polarizability of one particle used in expressions (5)-(7). Contrary to the MG model, the screening parameter in our case is decreased in the first step by the self-action via the substrate on the value $\left(e^{2} / 24\right)\left(\left(\varepsilon_{s}-\varepsilon_{a}\right) /\left(\varepsilon_{s}+\varepsilon_{a}\right)\right)$ for longitudinal and doubled value for transverse component. $e$ is the eccentricity and $\varepsilon_{a}$ and $\varepsilon_{s}$ are dielectric functions for the ambient and substrate correspondingly. This value is about $0.016 e^{2}$ in our case for the longitudinal component. In the second step, it is decreased by the value $n k_{0}^{2} G$, accounting interparticle interactions. If we have to take into account the size effect, it should be included into the particle polarizability in the beginning of calculations before the consideration of the interaction with the surface.

On the base of the obtained polarizability, it is possible in one or other way $[36,37]$ to calculate reflection coefficients. With the known Green function for half-space (e.g., [37]), we obtain

$$
\begin{aligned}
R_{s} & =\bar{R}_{0 s}+2 \pi i n X_{y y} \frac{k_{0}}{\sqrt{\varepsilon_{a}} \cos \vartheta}\left(1+\bar{R}_{0 s}\right)^{2}=\bar{R}_{0 s}+2 \pi i \\
& \cdot \frac{n \alpha_{\|}\left(k_{0} / \sqrt{\varepsilon_{a}} \cos \vartheta\right)\left(1+\bar{R}_{0 s}\right)^{2}}{1-n \alpha_{\|}\left(k_{0} / \sqrt{\varepsilon_{a}} \cos \vartheta\right)\left(1+\bar{R}_{0 s}\right)}, \\
R_{p} & =\bar{R}_{0 p} \\
& -\frac{2 \pi i}{D}\left[n \alpha_{\|} \frac{k_{0} \cos \vartheta}{\sqrt{\varepsilon_{a}}}\left(1-\bar{R}_{0 p}\right)^{2}-n \alpha_{\perp} \frac{k_{0} \sin ^{2} \vartheta}{\sqrt{\varepsilon_{a}} \cos \vartheta}\right],
\end{aligned}
$$




$$
\begin{aligned}
D & =\left[1+2 \pi i n \alpha_{\perp} \frac{k_{0} \sin ^{2} \vartheta}{\sqrt{\varepsilon_{a}} \cos \vartheta}\left(1+\bar{R}_{0 p}\right)\right] \\
& \cdot\left[1+2 \pi i n \alpha_{\|} \frac{k_{0} \cos \vartheta}{\sqrt{\varepsilon_{a}}}\left(1-\bar{R}_{0 p}\right)\right]+4 \pi^{2} n^{2} \alpha_{\|} \alpha_{\perp} \\
& \cdot \frac{k_{0}^{2} \sin ^{2} \vartheta}{\varepsilon_{a}} \bar{R}_{0 p}^{2},
\end{aligned}
$$

where $\bar{R}_{0}$ denotes reflection coefficients for the clean surface for corresponding polarization multiplied by phase shift produced by the separation from the substrate $e^{-2 k_{0} \cos 9 h}, h$ is the vertical size of particles, $\vartheta$ is the angle of incidence, $\varepsilon_{a}$ is the dielectric function of the ambient, and $k_{0}=2 \pi / \lambda$, where $\lambda$ is the wavelength as $\alpha_{\|}$and $\alpha_{\perp}$ are initial polarizabilities of one separated particle with the account of self-actions due to the interaction with the substrate and size correction.

This means that the layer of particles modifies the reflection as the layer with effective polarizabilities of the unit area $n X_{y y}$ along $y$-axis, $n \alpha_{\|} / D$ along $x$-axis, and $n \alpha_{\perp} / D$ along $z$ axis.

Proposed formalism is the first step for the description of optical properties of disordered nanostructured layers. Further averaging on the size, shape, and orientation is necessary like it was performed in [38].

\section{Conclusions}

We demonstrate that standard EMA models do not account direct interparticle interactions, which are important in the case of layers of nanoparticles. This deficiency does not allow MG model to fit presented experimental results, which clearly demonstrate the sensitivity of ellipsometry to interparticle interactions.

The presence of the shift of the resonance with increasing of the material amount in the film clearly indicates the necessity to account the direct interparticle interaction in the data interpretation, which is omitted in any standard effectivemedium approximation due to their three-dimensional background.

The formalism of Green function in $k-z$ representation was developed to describe the response of a layer of nanoparticles on the external illumination. The proposed formalism is the two-dimensional analogue of EMA. It uses the same very general parameters as standard EMA models and gives the explicit connection between these parameters and resulting optical properties of a layer of randomly distributed interacting nanoparticles.

\section{Conflict of Interests}

The authors declare that there is no conflict of interests regarding the publication of this paper.

\section{Acknowledgments}

The authors are very thankful to Dr. U. C. Fisher for the preparation of samples. E. G. Bortchagovsky and T. O. Mishakova acknowledge a guest scholarship from the Institute of Physics of the AV ČR.

\section{References}

[1] C. Brosseau, "Modelling and simulation of dielectric heterostructures: a physical survey from an historical perspective," Journal of Physics D: Applied Physics, vol. 39, no. 7, pp. 1277-1294, 2006.

[2] D. E. Aspness, "Local-field effects and effective-medium theory: a microscopic perspective," American Journal of Physics, vol. 50, pp. 704-708, 1982.

[3] D.-S. Wang and C.-W. Lin, "Density-dependent optical response of gold nanoparticle monolayers on silicon substrates," Optics Letters, vol. 32, no. 15, pp. 2128-2130, 2007.

[4] E. C. Chan and J. P. Marton, "Generalized Maxwell Garnett equations for rough surfaces," Journal of Applied Physics, vol. 45, no. 11, pp. 5004-5007, 1974.

[5] E. G. Bortchagovsky, V. Z. Lozovski, and T. O. Mishakova, "Model for the effective medium approximation of nanostructured layers with the account of interparticle interactions," in Metamaterials VI, vol. 8070 of Proceedings of SPIE, Prague, Czech Republic, May 2011.

[6] B. N. J. Persson, "Lateral interactions in small particle systems," Journal de Physique Colloques, vol. 44, no. 10, supplement 12, pp. 409-420, 1983.

[7] A. Liebsch and B. N. J. Persson, "Optical properties of small metallic particles in a continuous dielectric medium," Journal of Physics C: Solid State Physics, vol. 16, no. 27, pp. 5375-5391, 1983.

[8] R. G. Barrera, G. Monsivais, and W. L. Mochãn, "Renormalized polarizability in the Maxwell Garnett theory," Physical Review $B$, vol. 38, no. 8, pp. 5371-5379, 1988.

[9] A. Modinos, V. Yannopapas, and N. Stefanou, "Scattering of electromagnetic waves by nearly periodic structures," Physical Review B-Condensed Matter and Materials Physics, vol. 61, no. 12, pp. 8099-8107, 2000.

[10] J. I. Gittleman and B. Abeles, "Comparison of the effective medium and the Maxwell-Garnett predictions for the dielectric constants of granular metals," Physical Review B, vol. 15, no. 6, pp. 3273-3275, 1977.

[11] P. Sheng, "Pair-cluster theory for the dielectric constant of composite media," Physical Review B, vol. 22, no. 12, pp. 63646368, 1980.

[12] E. G. Bortchagovsky, "Ellipsometric method for investigation of the optical anisotropy of thin films: theory and calculations," Thin Solid Films, vol. 307, no. 1-2, pp. 192-199, 1997.

[13] S. Yamaguchi, "Theory of the optical properties of very thin inhomogeneous films," Journal of the Physical Society of Japan, vol. 17, no. 1, pp. 184-193, 1962.

[14] T. Yamaguchi, S. Yoshida, and A. Kinbara, "Optical properties of aggregated silver films," Journal of the Optical Society of America, vol. 61, no. 1, pp. 62-69, 1971.

[15] T. Yamaguchi, S. Yoshida, and A. Kinbara, "Optical effect of the substrate on the anomalous absorption of aggregated silver films," Thin Solid Films, vol. 21, no. 1, pp. 173-187, 1974.

[16] T. Yamaguchi, S. Yoshida, and A. Kinbara, "Effect of retarded dipole-dipole interactions between island particles on the optical plasma-resonance absorption of a silver-island film," Journal of the Optical Society of America, vol. 64, no. 11, pp. 1563-1568, 1974. 
[17] S. Giordano, "Effective medium theory for dispersions of dielectric ellipsoids," Journal of Electrostatics, vol. 58, no. 1-2, pp. 59-76, 2003.

[18] A. V. Goncharenko, V. Z. Lozovski, and E. F. Venger, "Effective dielectric response of a shape-distributed particle system," Journal of Physics Condensed Matter, vol. 13, no. 35, pp. 82178234, 2001.

[19] Y. Battie, A. En Naciri, W. Chamorro, and D. Horwat, "Generalized effective medium theory to extract the optical properties of two-dimensional nonspherical metallic nanoparticle layers," Journal of Physical Chemistry C, vol. 118, no. 9, pp. 4899-4905, 2014.

[20] G. Mie, "Beiträge zur Optik trüber Medien, speziell kolloidaler Metallösungen," Annalen der Physik, vol. 330, no. 3, pp. 377-445, 1908.

[21] W. T. Doyle, "Optical properties of a suspension of metal spheres," Physical Review B, vol. 39, no. 14, pp. 9852-9858, 1989.

[22] R. Ruppin, "Evaluation of extended Maxwell-Garnett theories," Optics Communications, vol. 182, no. 4, pp. 273-279, 2000.

[23] U. Kreibig and C. V. Fragstein, "The limitation of electron mean free path in small silver particles," Zeitschrift für Physik, vol. 224, no. 4, pp. 307-323, 1969.

[24] L. B. Scaffardi and J. O. Tocho, "Size dependence of refractive index of gold nanoparticles," Nanotechnology, vol. 17, no. 5, pp. 1309-1315, 2006.

[25] G. Bosi and B. De Dormale, "Substrate-related effects on the optical behavior of a granular surface: the Maxwell Garnett theory revisited," Journal of Applied Physics, vol. 58, no. 1, pp. 513-517, 1985.

[26] D. J. Bergman, "The dielectric constant of a composite material-a problem in classical physics," Physics Reports, vol. 43, no. 9, pp. 377-407, 1978.

[27] D. Bedeaux and J. Vlieger, Optical Properties of Surfaces, Imperial College Press, London, UK, 2004.

[28] T. W. H. Oates, M. Ranjan, S. Facsko, and H. Arwin, "Highly anisotropic effective dielectric functions of silver nanoparticle arrays," Optics Express, vol. 19, no. 3, pp. 2014-2028, 2011.

[29] M. Lončarić, J. Sancho-Parramon, and H. Zorc, "Optical properties of gold island films-a spectroscopic ellipsometry study," Thin Solid Films, vol. 519, no. 9, pp. 2946-2950, 2011.

[30] N. T. Gladkih, Ed., Surface Effects and Phase Transformations in Condensed Films, Kharkov State University, 2004 (Russian).

[31] E. G. Bortchagovsky, T. O. Mishakova, and K. Hingerl, "Ellipsometry of monolayers of metallic nanoparticles taking into account depolarization," Thin Solid Films, vol. 571, no. 3, pp. 625-630, 2014.

[32] http://www.jawoollam.com.

[33] E. David, "Deutung der Anomalien der optischen Konstanten dünner Metallschichten," Zeitschrift für Physik, vol. 114, no. 7-8, pp. 389-406, 1939.

[34] E. G. Bortchagovsky and U. C. Fischer, "On the modulation of optical transmission spectra of thin dye layers by a supporting medium," Journal of Chemical Physics, vol. 117, no. 11, pp. 53845392, 2002.

[35] M. Meier, A. Wokaun, and P. F. Liao, "Enhanced fields on rough surfaces: dipolar interactions among particles of sizes exceeding the Rayleigh limit," Journal of the Optical Society of America B, vol. 2, no. 6, pp. 931-949, 1985.

[36] E. G. Bortchagovsky, V. Z. Lozovski, and T. O. Mishakova, "Theory of the ellipsometry of a layer of semiconductor nanoparticles covering the substrate," Ukrainian Journal of Physics, vol. 55, no. 10, pp. 1135-1144, 2010.

[37] E. G. Bortchagovsky, O. M. Getsko, V. Z. Lozovski, and B. I. Khudik, "Ellipsometry of the ultrathing films," Optics and Spectroscopy (USSR), vol. 66, pp. 783-788, 1989.

[38] J. Toudert, L. Simonot, S. Camelio, and D. Babonneau, "Advanced optical effective medium modeling for a single layer of polydisperse ellipsoidal nanoparticles embedded in a homogeneous dielectric medium: surface plasmon resonances," Physical Review B, vol. 86, no. 4, Article ID 045415, 2012. 

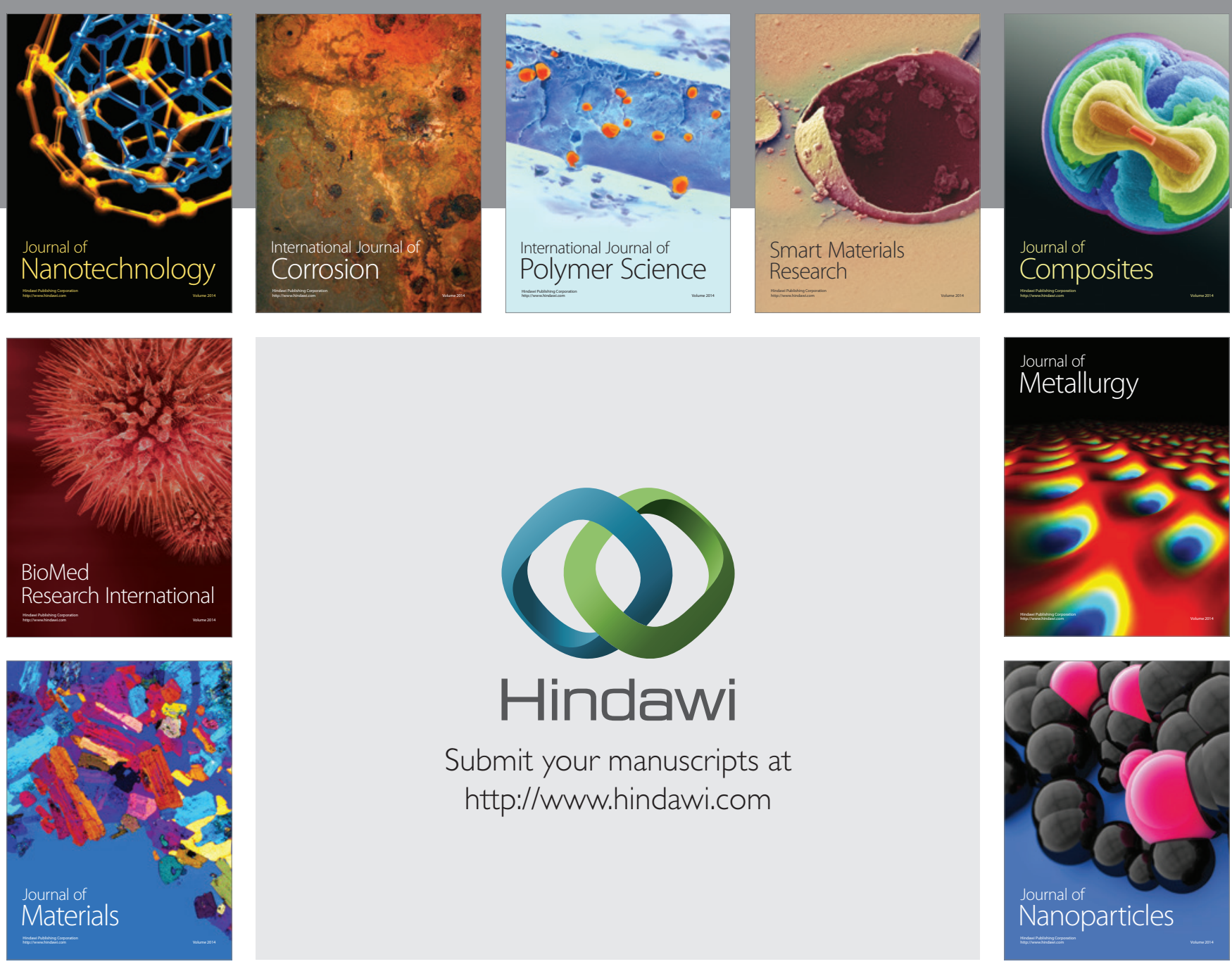

Submit your manuscripts at http://www.hindawi.com
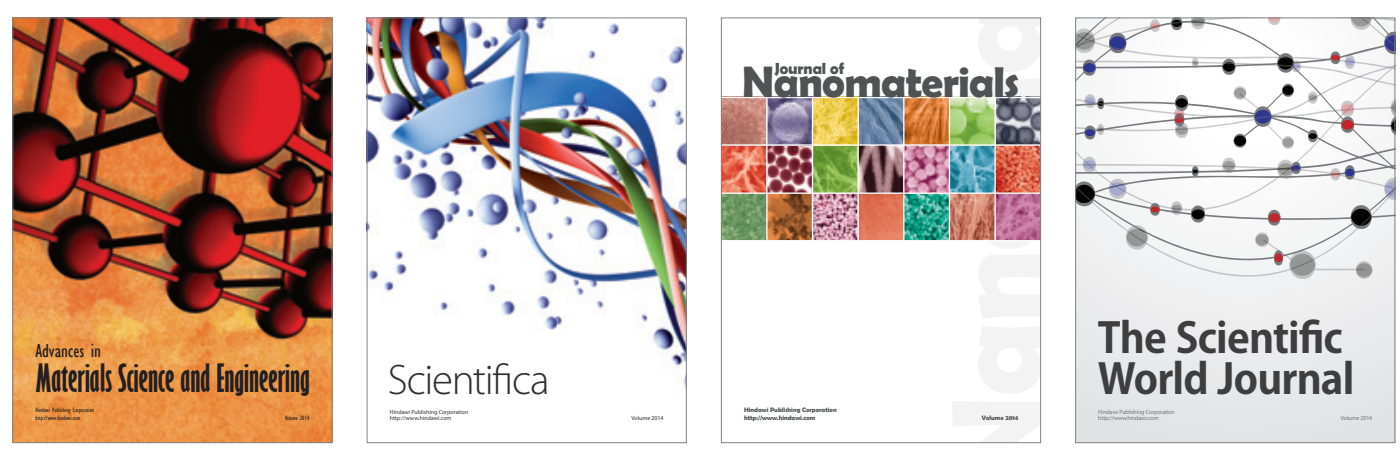

\section{The Scientific World Journal}
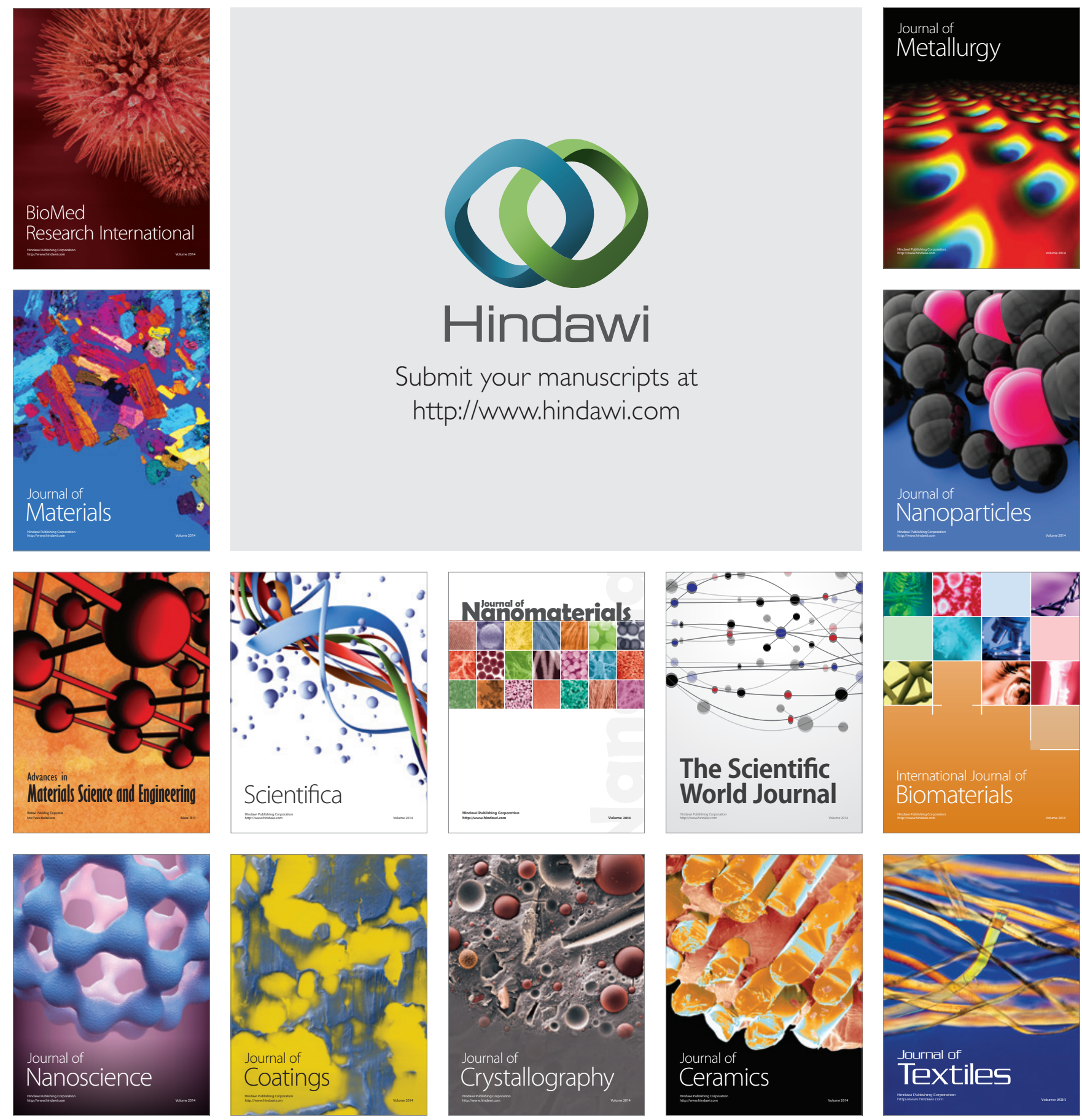